\title{
Comparison of Functional Outcome Between Computer Navigated Total Knee Replacement with Conventional Method
}

\author{
Ahmad Munir Hashim ${ }^{1}$, Mohd Shahidan b. Noor Rahin ${ }^{1}$, Ahmad Hafiz b. Zulkifly \\ ${ }^{1}$ Department of Orthopaedics, Traumatology and Rehabilitation, Kulliyyah of \\ Medicine, International Islamic University Malaysia
}

Presenter: Ahmad Munir Hashim

Introduction: Total knee replacement (TKR) is considered one of most successful treatment in treating knee arthritis. It aims at restoring neutral mechanical axis, balanced ligaments and normal $Q$ angle. Surgeons have been using manual jig to achieved perfect implant placement. Since 2002 computer assisted surgery (CAS) is gaining popularity in TKR surgery to improved accuracy of implant placement. In a large meta-analysis by Bauwen's et al, computer assisted surgery reduced number of patients with post-operative malalignment $>3$ degree. In another study by Kim et al found there was no no difference in alignment between computer assisted and conventional method TKR in bilateral TKR with one using conventional and the other computer assisted. In our study we compared the functional outcome between these two methods. Materials and Methods: This was a cross-sectional study conducted in Penang General Hospital comparing the functional outcome using SF-36 questionnaire and Hospital for Special Surgery (HSS) knee scoring. It involved 35 knees in each arm. All patients were operated by single surgeon using same type of implant and navigation system. Patients with post-operative complications were excluded. Results: We found that there was no significant difference $(p<0.05)$ in both SF-36 and HSS knee score using U Mann Whitney test. Conclusion: There was no difference in functional outcome between conventional TKR and CAS 